\title{
Dewetting-Driven Hierarchical Self-Assembly of Small Semiconducting Molecules
}

\author{
Jean-Nicolas Tisserant, ${ }^{\text {ab }}$ Roland Hany, ${ }^{\text {a }}$ Stefan Partel, ${ }^{\text {c }}$ Gian-Luca Bona, ${ }^{\text {def }}$ Raffaele \\ Mezzenga, ${ }^{b}$ Jakob Heier*a
}

${ }^{a}$ Empa, Laboratory for Functional Polymers, 8600 Dübendorf, Switzerland. E-mail: Jakob.Heier@empa.ch.

${ }^{\mathrm{b}}$ Swiss Federal Institute of Technology, Food and Soft Materials Science, Institute of Food, Nutrition and Health, 8092 Zürich, Switzerland

${ }^{\mathrm{c}}$ Vorarlberg University of Applied Sciences, Research Center for Microtechnology, 6850

Dornbirn, Austria

${ }^{\mathrm{d}}$ Empa, Swiss Federal Laboratories of Materials Science and Technology

8600 Dübendorf, Switzerland

${ }^{\mathrm{e}}$ Swiss Federal Institute of Technology, Department of Information Technology and Electrical Engineering, 8092 Zürich, Switzerland

${ }^{\mathrm{f}}$ Swiss Federal Institute of Technology, Laboratory for Photonic Materials and

Characterization, 1015 Lausanne, Switzerland

Correspondence to be addressed to:

Dr. Jakob Heier, Empa, Laboratory for Functional Polymers, 8600 Dübendorf, Switzerland: Jakob.Heier@empa.ch 


\section{Abstract}

We describe the self-organization of PCBM and a cyanine dye on chemically patterned surfaces during spin coating from solution. On homogeneous surfaces, a transient bilayer forms, which in a later stage decomposes into PCBM droplets in a matrix of the cyanine dye. On the patterned surface also a PCBM droplet phase develops, but the final film structure is greatly determined by contact line pinning of the PCBM domains to the substrate pattern. Three characteristic morphology regimes separated by wetting transitions were observed for different ratios between the natural domain dimensions and the underlying pattern periodicity. We demonstrate that contact line pinning can be an important means to control the film morphology in systems where films are coated from solution. This process can be exploited as a general and versatile method for patterning small semiconducting molecules into 1D and 2D photonic crystals.

Keywords: cyanines, fullerenes, thin films, liquid-liquid dewetting, phase transition 


\section{Introduction}

Emerging device applications in the field of organic electronics and optoelectronics require the control of organic thin film structures on sub-micron length scales. ${ }^{1}$ For instance, a crucial issue in the development of organic photovoltaic devices is the optimization of lightmatter interaction. 2D-photonic crystals with periodic patterns in the range of the visible light wavelength are promising candidates for enhancing light absorption through scattering, interference or in coupling of "slow Bloch modes". ${ }^{2}$ Such repeating structures of semiconductors are also suitable for the development of all-optical switches. ${ }^{3}$ However, the ability to organize organic nano- and micro-scale objects on surfaces to form defect-free 2-D photonic crystals following principles that are cheap and up-scalable remains challenging. Methods commonly used to manufacture such objects include top-down approaches based on lithography ${ }^{4,5}$ or inkjet printing. ${ }^{6}$ Alternatively, organic functional materials can be structured by self-assembly processes, such as pattern formation via polymer demixing of multicomponent blends or instabilities that develop during film formation. ${ }^{7}$ Among these bottomup approaches, liquid-liquid dewetting of blends of small semiconducting molecules is a promising method as it can generate hierarchical structures in a controllable manner. ${ }^{8}$

Micro-contact printing $(\mu \mathrm{CP})$ of thiol self-assembled monolayers (SAMs) on gold ${ }^{9,10}$ is a well described method to induce long-range order in a dewetting system on sub-micron scales. Wetting on chemically patterned surfaces for single layers and bilayers has been investigated both theoretically ${ }^{11,12}$ and experimentally. ${ }^{13,14}$ Phase separation of polymers and block copolymers on patterned substrates has been studied extensively. ${ }^{15,16}$ Self-assembly in evaporating systems has resulted in astonishing patterns originating from Marangoni instabilities, but has also proven to be difficult to control. ${ }^{17}$ In parallel, small semiconducting dyes are attracting increasing interest over the last few years, since their purification is relatively easy, they are mono-disperse and their tendency to aggregate confers them unique optical properties. ${ }^{18}$ For instance, cyanine dyes show nonlinear optical and electronic 
properties due to their ability to form nano-crystalline $\mathrm{H}$ - and J-aggregates on $[6,6]$-Phenyl $\mathrm{C}_{61}$-butyric acid methyl ester (PCBM) templates. ${ }^{19}$

We have shown earlier that a blend solution of a cyanine dye (CyC) and PCBM undergoes phase separation by liquid-liquid dewetting during spin coating on homogeneous, hydrophilic substrates. ${ }^{8}$ First, a transient bilayer is formed consisting of cyanine-rich solution in contact with the substrate and of PCBM-rich solution in contact with air. Upon solvent evaporation, the thinner of the two layers destabilizes and undergoes spinodal dewetting. Therefore, for off-critical solutions with a higher CyC fraction, PCBM lenses form that float on top of the dye layer. Typically, the dye layer underneath the PCBM lens also ruptures later on, leading to fully laterally separated PCBM domains in a dye matrix (Fig. 1a, c). For a higher PCBM fraction, the resulting morphology is inversed and consists of dye droplets in a matrix of PCBM (Fig. 1b, d). The dewetting process is characterized by a typical decomposition wavelength $\lambda$, which is proportional to the average thickness of the corresponding layer in the final film (Fig. 1e). The hole structure arises because PCBM will dewet from the dye droplets that are in contact with the substrate interface. ${ }^{8}$

Here, we apply the process of liquid-liquid dewetting of the CyC:PCBM blend solution to a substrate with a periodic surface energy pattern. This adds the periodicity $\pi$ of the pre-patterned substrate as a second relevant length scale to the system. The phenomenon of contact line pinning can guide the separating system into morphologies with a very high phase contrast.

\section{Results}

In a first experiment 1:1 molar blend solutions of $\mathrm{CyC}: \mathrm{PCBM}$ in chlorobenzene were spin coated onto patterned surfaces such that the characteristic morphology length scale $\lambda$ was smaller than the periodicity $\pi$ of the patterned surface. In this case, the droplet morphology 
that developed on a homogeneous hydrophilic substrate (Fig. 1a) was maintained also across the patterned surface, but with a larger average PCBM droplet size on the $\mathrm{CH}_{3}$-terminated SAM (Fig. 2a). Selective dissolution of PCBM showed that PCBM droplets wetted the substrate at the positions of the SAMs, while PCBM on the hydrophilic gold lines remained fully embedded in the cyanine matrix (Fig. 2b). A Fourier transform of the SPM image revealed the periodic structure as well as the droplet size distribution (Fig. 2d). Similarly to the situation on a homogeneous gold substrate (Fig. 1b), blend films coated from 1:2 molar CyC:PCBM solutions preserved the hole morphology across the entire surface of a structured sample (Fig. 2e). Again, a two population pattern developed (Fig. 2e and 2f), and the system was comprised of cyanine droplets in a PCBM matrix with larger droplets on the gold lines. A direct comparison with the morphology that would form on a homogeneous SAM was not possible, as solutions dewetted from the substrate during spin coating completely.

Given the symmetry of the final morphologies, we focus in the following on the situation where PCBM formed droplets in a cyanine matrix. We study the behavior of the system for different ratios of the characteristic decomposition wavelength $\lambda$ to the patterning periodicity $\pi$. This can practically be studied by either varying the film thickness for a fixed pattern periodicity (varying $\lambda$ and keeping $\pi$ constant), or by varying the pattern periodicity at constant film thickness (varying $\pi$, constant $\lambda$ ). We here follow the first strategy and considered films obtained from a 1:1 molar CyC:PCBM solution. As the relevant variable is the droplet size and not the film thickness, we describe the data as a function of the natural decomposition wavelength obtained from the Fourier-Transforms of films on non-patterned hydrophilic substrates (Fig. 1e).

Fig. 3 shows the response of films of varying thicknesses and thus varying droplet sizes to the same surface pattern. Three thickness regimes characterized by a specific morphology could be identified. An under-resonance morphology was observed for thin films where the blend system provides a characteristic decomposition wavelength smaller than the 
substrate geometry's characteristic size, $\lambda<<\pi$ (Fig. 3a). This regime has already been described in the previous section. A resonance morphology was observed when the system's characteristic decomposition wavelength approached the periodicity of the patterned substrate, $\lambda \leq \pi$ (Fig. 3b). In this regime the SAM lines were fully covered by PCBM and the gold surface was covered by a dye matrix with embedded PCBM droplets. An over-resonance morphology was observed for even thicker films. In this case, the characteristic decomposition wavelength of the system was larger than the pattern periodicity, $\lambda>\pi$ (Fig. 3c). Also here the SAM lines were predominantly covered by PCBM, but the cylinders broke again into discrete droplets aligned along the pattern line direction, and the domains became wider than the SAM stripe. At the same time, the gold lines were getting increasingly depleted from PCBM. In rare cases we observed coalescence of domains originating from neighboring SAM stripes (Fig. 3d). Increasing the film thickness further (Fig. 3e) resulted in the most extreme form of that morphology classification. Surprisingly, the PCBM lens shape was then again circular and the whole substrate was covered with a nearly cubic lattice arrangement of droplets having exactly the sizes corresponding to the pattern periodicity (Fig. 3f). These domains were much smaller compared to PCBM droplets that would have formed on a homogeneous substrate.

Another way of characterizing the different regimes is in terms of the differences in PCBM lens sizes on the two different surfaces. While in the under-resonant regime the lenses only differ slightly in size, the difference goes towards infinity for the resonant and overresonant regime. A ratio of infinity represents the situation that the gold stripes are fully depleted from PCBM. These statements are quantified in Fig. 4, where all relevant lateral length scales are plotted versus the natural decomposition wavelength of the PCBM domains on plain gold. For all thicknesses, the droplets that formed on the gold stripes were smaller than the droplets forming on a homogeneous gold substrate. For small droplet sizes this deviation was only minor, but was getting significant for larger droplets. This reflects the fact 
that with increasing droplet size, PCBM lenses on the SAM grow at the expense of the lenses on top of the gold surface until they fully disappear and are displaced by PCBM lenses originating from the SAM. For droplets forming on the SAM lines, we define two length scales, the droplet dimension perpendicular and parallel to the grating direction. The two values coincide until a critical natural decomposition wavelength $\lambda_{\mathrm{cr}} \approx 750 \mathrm{~nm}$, consistent with the observations that up to a certain size circular domains are observed. When the natural wavelength approaches the periodicity of the grating, the two curves separate, corresponding to an elongation of the domains along the grating while the domains size perpendicular to the grating remains almost constant. At full SAM coverage by PCBM, the value of the parallel component reaches infinity (not shown in Fig. 4). For increasing feature dimensions, the PCBM stripes on the SAM break up into individual domains. Finally, the lines representing the domain dimensions parallel and perpendicular to the grating merge and asymptotically approach the line representing the grating periodicity. The domains are thus pinned to the underlying grating (Fig. 3f).

So far, we showed how a CyC:PCBM blend film responds to a surface energy pattern by varying the film thickness for a fixed substrate periodicity. Alternatively, the case was studied where the film thickness was constant and the periodicity of the underlying pattern was varied. Qualitatively, the same results were observed. We show in Fig. 5 SPM scans of one particular example where only the gold stripe width was varied, while the SAM width remained constant, displaying the transition between under-resonance and resonance regime in more details. Films coated from a 1:1 molar $\mathrm{CyC}$ :PCBM solution had a natural wavelength of $\lambda=1.7 \mu \mathrm{m}$, corresponding to an average PCBM thickness of $\mathrm{d}_{\mathrm{PCBM}}=120 \mathrm{~nm}$. For the lower pattern periodicity (Fig. 5a), no dye remains on the SAM and the SAM stripes are fully covered by PCBM (Fig. 5d). On the gold stripe, one line of PCBM lenses remains embedded in the dye matrix. This corresponds to the near-resonance case, as also shown in Figure 3b and compiled in Fig. 4. Here the PCBM domains along the grating are infinitively long. 
Increasing the gold width to $6 \mu \mathrm{m}$ and $8 \mu \mathrm{m}$ shows the transition from the resonant to the under-resonance morphology. The PCBM stripe gets disrupted (Fig. 5b,c) because of dye remaining on the SAM stripe (Fig. 5e). The mechanism is similar to the formation of the perforated PCBM morphology shown in Fig. 1b, as PCBM dewets from CyC. At the same time the ratio of PCBM to the dye on the gold stripe increases, leading to a transformation from isolated droplets to an interconnected network. Effectively, the contrast of the morphology from substrate to substrate gets smaller, which is a characteristic of the underresonant morphology.

\section{Discussion}

With the help of Fig. 6 we introduce a comprehensive model that explains our observations. Similar to the homogeneous case, also on a heterogeneous substrate a transient bilayer with the PCBM rich phase at the air interface and the $\mathrm{CyC}$ rich phase at the substrate interface develops during film formation. The solution senses an average surface energy of the patterned substrate. The surface energies of CyC $(\gamma=34.3 \mathrm{mN} / \mathrm{m})$ and $\operatorname{PCBM}(\gamma=23.9$ $\mathrm{mN} / \mathrm{m}$ ) have been measured before. ${ }^{8}$ For the macroscopic surface energy of the patterned surface, a value of $28.9 \pm 2 \mathrm{mN} / \mathrm{m}$ was obtained from contact angle measurements. This is somewhat higher than the value reported for a pure $\mathrm{CH}_{3}$-terminated SAM on $\mathrm{Au}(\gamma=19-20$ $\mathrm{mN} / \mathrm{m}) .{ }^{20}$ The initial surface energy of neat (111) Au can be extremely high $(\gamma=1250$ $\mathrm{mN} / \mathrm{m}),{ }^{21}$ but this value rapidly decreases due to contaminations when the surface is exposed to air. $^{22}$

We assume that also the next stage in the morphology evolution, the break-up of the PCBM layer into lenses floating on a liquid dye phase, develops on the patterned surface similarly as it does on a homogeneous surface (Fig. 6a). Direct evidence for this process is the final morphology observed in the under-resonance regime (Fig. 2a). To understand at which 
stage of solvent evaporation the PCBM lenses develop, it is instructive to inspect the thickness evolution of the evaporating film and to compare that with the in-plane time evolution of the PCBM lens dimension.

Film thinning was measured with a laser beam reflecting from the solution and substrate interfaces during spin coating, following the procedure reported in previous literature. ${ }^{23}$ The time evolution of the lateral domains was determined from the PSD-peaks of samples where the solidification times were varied by the temperature inside the spin coating chamber, while all other parameters were kept constant.

Figure 7 summarizes the results of these experiments. In the relevant film formation time window between $9 \mathrm{~s}$ and $15 \mathrm{~s}$, the characteristic wavelength of the PCBM lenses does not change significantly. Irrespective of the coating speed, the film thickness decreased almost linearly in about $5 \mathrm{~s}$ from $5 \mu \mathrm{m}$ to the final thickness, indicative for film thinning by solvent evaporation. It is therefore reasonable to assume that during the last seconds of film formation the wavelength of the PCBM droplet pattern of the film is maintained.

We also know that the natural decomposition wavelength depends linearly on the final film thickness, from here we must assume that the PCBM layer breaks into droplet phases at a certain solution concentration. Together with the findings from before, we can postulate that the final film is formed by a sequence of droplet formation, followed by flattening into PCBM lenses due to solvent evaporation perpendicular to the film surface. This is an important finding as it assures us that the framework is given that surface patterning can induce meaningful ordering in the samples.

PCBM lens ordering on the patterned substrate then occurs in the last stage of solvent evaporation when the PCBM lenses get in close vicinity to the substrate. The relevant processes are depicted in Fig. 6. PCBM has a high affinity for the SAM, while the affinity of PCBM to the gold surface is unfavorable. When the PCBM lenses are small compared to the stripe pattern, the lenses will cover the SAM surface under a low contact angle (Fig. 6b), 
while PCBM will avoid the contact with the gold surface. For the same volume of PCBM lenses on both substrates, the domains on the SAM should then be larger in diameter but smaller in height compared to the domains on gold. This is indeed what we observed (Fig. 2c). The exact shape of the domains is determined by the contact lines at the substrate - PCBM $\mathrm{CyC}$ interface as well as the PCBM - CyC - air interface and can take a rather complex form. Young's equation combined with the approach of Good and Girifalco ${ }^{24}$ to approximate the interfacial tensions between two compounds from the individual surface energies yields a contact angle of $\Theta_{\mathrm{SAM}}=35^{\circ}$ for PCBM on the SAM. This is the value we also obtained experimentally for an isolated PCBM lens on the SAM. The calculation from above actually failed in describing the contact angle of PCBM in a dye matrix correctly, reflecting the more complex lens shape.

By increasing the film thickness a regime is entered where the dimensions of the PCBM lenses get comparable to the pattern stripe width. In that case the border between the SAM and gold results in an immobilization of the contact line where PCBM touches the substrate. This "contact line pinning" has strong implications for pattern formation: ${ }^{25}$ Increasing the droplet volume further will lead to anisotropic domain growth until the SAM stripe is fully wetted by a PCBM layer. Fig. 5a for example shows a SAM stripe fully covered by a relatively thin PCBM layer. A further increase in droplet volume will lead to an increase of the PCBM contact angle at the pinned contact line. The particular feature of this resonance regime is that the PCBM contact angle at the interface can take any value between $\Theta_{\mathrm{SAM}}<$ $\Theta<\Theta_{\mathrm{AU}}$ (Fig. 6c).

For even thicker films the over-resonant regime is entered. This regime is characterized by sufficiently large domains where the droplets have reached a contact angle $\Theta=\Theta_{\mathrm{AU}}$. The contact line will then move onto the gold stripe (Fig. 6d). An SPM image of this regime is shown in Fig. 3c, where the width of the PCBM domains is by far exceeding the width of the SAM stripe. The contact angles of PCBM on the different substrates and two 
examples for the case of contact line pinning obtained from cross-sections from SPM scans are shown in Fig. 6e. With the $\mathrm{CH}_{3}$-terminated SAM and gold, PCBM forms a contact angle of $\Theta=35^{\circ}$ and $\Theta=125^{\circ}$, respectively. We can here also observe that the PCBM lenses originating from different stripes show coalescence (Fig. 3d). In each of the three morphology regimes, the PCBM lens morphology is in a different wetting state, the morphologies are separated by so-called wetting transitions, visualized in Fig. $6 .^{26}$

In the over-resonant regime the cylindrical PCBM segments break up again into elongated domains stretched along the SAM stripe. We can explain this observation with another capillary instability. The classical Rayleigh-Plateau instability refers to a surface tension induced instability of a cylindrical liquid column. ${ }^{27}$ Work of Gau et al. demonstrated that a liquid on a stripe of hydrophilic material forms a single large buldge, as result of a Rayleigh instability of infinite destabilization wavelength. ${ }^{13}$ A linear stability analysis of the same system showed that cylindrical segments are getting capillary unstable when the contact angle reached $\theta=90^{\circ} .{ }^{28}$ We observed the transition from an infinite long cylinder to elongated domains between contact angles of $\theta=65^{\circ}$ and $\theta=125^{\circ}$. Similarly, in the regime of the Rayleigh-Plateau instability, the most unstable wavelength is of the order of the cylinder radius. For the sample shown in Figure $3 \mathrm{f}$ a typical droplet size of $\lambda=1.52 \mu \mathrm{m}$ was measured, which is close to the pattern periodicity of $\pi=1.50 \mu \mathrm{m}$.

\section{Experimental}

\section{Preparation of patterned substrates}

Clean gold surfaces were manufactured by low pressure metal deposition on silicon wafers or glass. Metal pellets were purchased from Cerac Inc. A $3 \mathrm{~nm}$ thick chromium layer and a 30 $\mathrm{nm}$ thick gold layer were deposited at a typical deposition rate of $0.1 \mathrm{~A} \mathrm{~s}^{-1}$ at a starting pressure of $6 \times 10^{-6}$ mbar. To allow optical measurements in transmission mode, some semi- 
transparent samples were also prepared on glass substrates. In this case, the thicknesses of chromium and gold were $3 \mathrm{~nm}$. Patterned poly(dimethylsiloxane) stamps (PDMS) were prepared by pouring a vacuum-degassed 10:1 (wt:wt) base:curing-agent mixture (Sylgard 184-Dow Corning) on a pre-patterned silicon wafer bearing $280 \mathrm{~nm}$ deep gratings with different periodicities and the system was then cured at room temperature for $24 \mathrm{~h}$. The patterned PDMS stamp was swollen for $5 \mathrm{~s}$ by drop contact with a $0.5 \mathrm{mM}$ solution of octadecanethiol (Sigma Aldrich) and was then dried under nitrogen flow for $10 \mathrm{~s}$. It was then maintained in contact with a freshly deposited gold surface for $10 \mathrm{~s}$, resulting in a stripe pattern of $\mathrm{CH}_{3}$-terminated SAMs (hydrophobic) on the hydrophilic gold surface. Patterned substrates were characterized by Frictional Force Microscopy and Scanning Electron Microscopy.

\section{Film formation}

The cyanine dye 1,10-diethyl-3,3,3',3'-tetramethylcarbocyanine perchlorate (CyC) was synthesized in our laboratory. ${ }^{29}[6,6]-$ Phenyl $\mathrm{C}_{61}$-butyric acid methyl ester (PCBM) was purchased from Solenne B.V., The Netherlands. PCBM and CyC were dissolved in the common solvent chlorobenzene and were then spin coated onto patterned or homogeneously coated gold substrates. All experiments were performed at room temperature. Thicknesses were adjusted by varying the spin speed. PCBM was selectively removed from the blend films by immersing the sample into hexane under sonication, and the dye was selectively removed from the blend films by immersing the sample in 2,2,3,3-tetrafluoropropanol.

\section{Characterization}

The morphology of the samples was measured by Scanning Probe Microscopy (SPM) on a Nanosurf Mobile S in tapping mode at a resonance frequency of $170 \mathrm{kHz}$. We used rectangular silicon cantilevers (Mikromasch, Nanosensors) with a typical force constant of 40 
$\mathrm{N} / \mathrm{m}$ and a tip radius of curvature of $10 \mathrm{~nm}$. Images were analyzed with the WsXM scanning probe microscopy software. ${ }^{30}$ Final average film thicknesses were determined with UV-vis spectroscopy as described in ref. 8. The surface energy of the patterned substrate was determined from the advancing contact angles of droplets of water, glycerol, hexane and diiodomethane. ${ }^{31}$

\section{Conclusion}

We gave experimental evidence for wetting transitions between different morphology regimes that can be found in a blend film of small molecules deposited from solution onto an energy patterned surface. In one regime, evaporating PCBM droplets were pinned to the surface pattern, allowing for precise deposition of molecules onto a defined surface area. This process can have important implications for generating structured surfaces directly from solution by spin coating.

We only considered two blend compositions and one energy pattern contrast. Given the huge parameter space, we expect that a much larger variety of structures can be manufactured, especially in the regime where contact-line pinning occurs.

\section{Acknowledgements}

This work was supported by the Swiss National Science Foundation (SNF) under grant number 200021_132502. We acknowledge T. Geiger for dye synthesis, and Ü. Müller and the Swiss Scanning Probe Microscopy User Laboratory at Empa for SPM support. We thank J. Groenewold (University of Utrecht) for helpful discussions. 


\section{References}

1 Nanostructured Conductive Polymers, (Ed: A. Eftekhari), John Wiley \& Sons Ltd, West Sussex, United Kingdom, 2010.

2 D. Duché, L. Escoubas, J.-J. Simon, P. Torchio, W. Vervisch and F. Flory, Appl. Phys. Lett., 2008, 92, 193310.

3 V. R. Almeida, C. A. Barrios, R. R. Panepucci and M. Lipson, Nature, 2004, 431, 1081-1084.

4 E. Menard, M. A. Meitl, Y. Sun, J. U. Park, D. J. L. Shir, Y. S. Nam, S. Jeon and J. A. Rogers, Chem. Rev., 2007, 107, 1117-1160.

5 G. M. Wallraff and W. D. Hinsberg, Chem. Rev., 1999, 99, 1801-1821.

6 H. Sirringhaus, T. Kawase, R. H. Friend, T. Shimoda, M. Inbasekaran, W. Wu and E.

P. Woo, Science, 2000, 290, 2123-2126.

7 R. V. Craster and O. K. Matar, Rev. Mod. Phys., 2009, 81, 1131-1198.

8 J. Heier, J. Groenewold, S. Huber, F. Nüesch and R. Hany, Langmuir, 2008, 24, 73167322.

9 A. Kumar and G. M. Whitesides, App. Phys. Lett., 1993, 63, 2002-2004.

10 J. C. Love, L. A. Estroff, J. K. Kriebel, R. G. Nuzzo and G. M. Whitesides, Chem. Rev., 2005, 105, 1103-1169.

11 S. D. Iliev and N. Ch. Pesheva, Langmuir, 2003, 19, 9923 - 9931.

12 D. Bandyopadhyay, A. Sharma and C. Rastogi, Langmuir, 2008, 24, 14048-14058.

13 H. Gau, S. Herminghaus, P. Lenz and R. Lipowsky, Science, 1999, 283, 46-49.

14 J. Léopoldès and P. Damman, Nat. Mat., 2006, 5, 957-961.

15 M. Böltau, S. Walheim, J. Mlynek, G.Krausch and U. Steiner, Nature, 1998, 391, 877879.

16 J. Heier, E. J. Kramer, S. Walheim and G. Krausch, Macromolecules, 1997, 30, 66106614. 
17 H. Mancini and D. Maza, Europhys. Lett., 2004, 66, 812-818.

18 F. C. Spano and S. Mukamel, J. Chem. Phys., 1991, 95, 7526-7540.

19 J. Heier, R. Steiger, F. Nüesch and R. Hany, Langmuir, 2010, 26, 3955-3961.

20 J. Genzer and E. J. Kramer, Phys. Rev. Lett., 1997, 78, 4946-4949.

21 R. J. Needs and M. Mansfield, J. Phys.: Condens. Mat., 1989, 1, 7555-7563.

22 F. E. Bartell and J. T. Smith, J. Phys. Chem., 1953, 57, 165-172.

23 P. C. Jukes, S. Y. Heriot, J. S. Sharp and R. A. L. Jones, Macromolecules, 2005, 38, 2030-2032.

24 D. Myers, Surfaces, Interfaces and Colloids, Wiley-VCH, New York, 1999.

25 S. Herminghaus, M. Brinkmann and R. Seemann, Annu. Rev. Mater. Res., 2008, 38, 101-121.

26 R. Lipowsky, Cur. Op. Coll. Int. Sci., 2001, 6, 40-48.

27 J. Eggers, Rev. Mod. Phys., 1997, 69, 865-929.

28 R. L. Speth and E. Lauga, New J. Phys., 2009, 11, 075024.

29 L. A. Ernst, R. K. Gupta, R. B. Mujumdar and S. Waggoner, Cytometry, 1989, 10, 3.

30 I. Horcas, R. Fernández, J. M. Gómez-Rodríguez, J. Colchero, J. Gómez-Herrero and A. M. Baro, Rev. Sci. Instrum., 2007, 78, 013705.

31 D. K. Owens and R. C. Wendt, J. Appl. Polym. Sci., 1969, 13, 1741. 

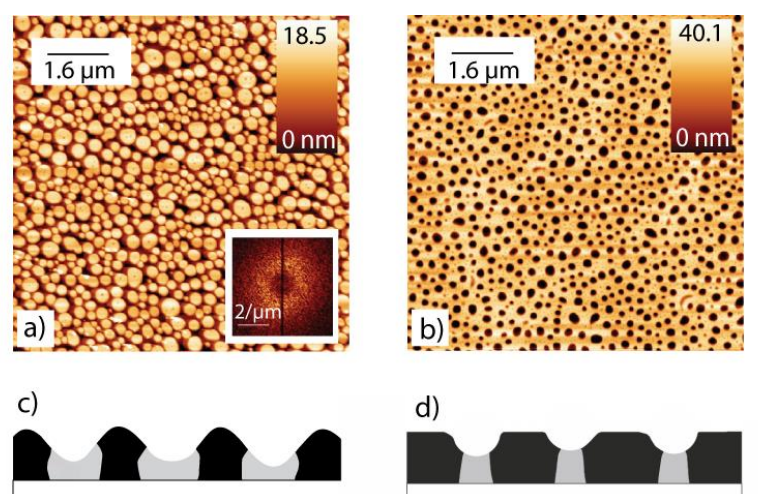

d)

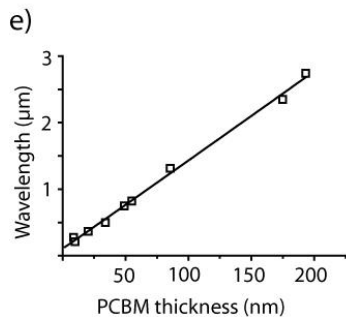

f)
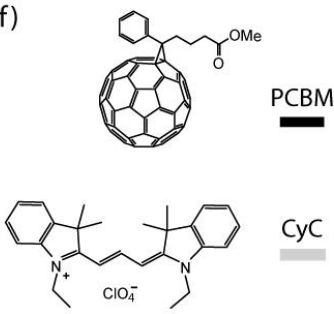

Fig. 1 (a) SPM image of PCBM droplets embedded in a matrix of CyC dye. (b) SPM image of a perforated PCBM film; the sinkholes contain CyC droplets; films (a) and (b) were coated on a homogeneously gold-coated silicon wafer. (c, d) Schematic drawings of the film morphologies in (a) and (b), respectively. (e) Scaling plot of the characteristic decomposition wavelength vs. average PCBM film thickness. Data are reproduced with permission from [8], copyright 2008 American Chemical Society. (f) Structure formulas of PCBM and the cyanine dye CyC. 

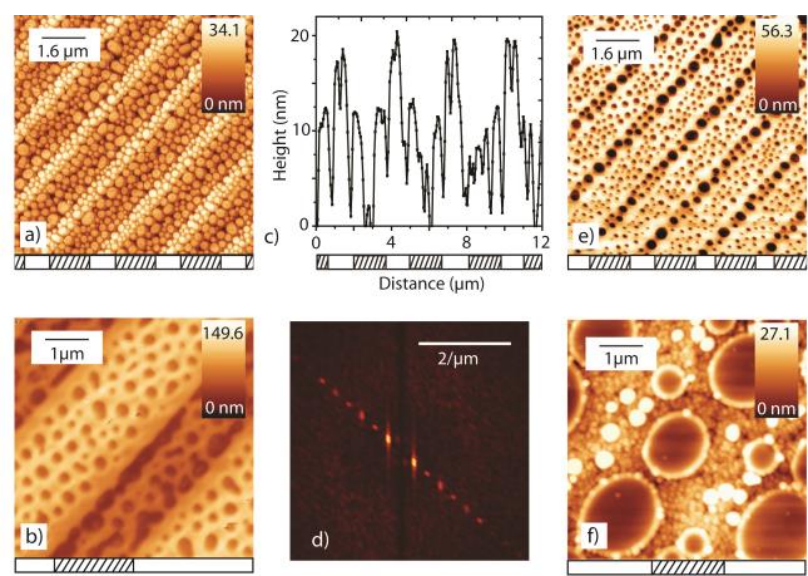

Fig. 2 SPM images of films on patterned substrates. The pattern consisted of alternating stripes of $1.5 \mu \mathrm{m}$ wide $\mathrm{CH}_{3}$-terminated SAM lines and $1.0 \mu \mathrm{m}$ wide gold lines for (a), (e) and (f), and of $1.0 \mu \mathrm{m}$ wide SAM lines and $1.5 \mu \mathrm{m}$ wide gold lines for (b); the striped rectangles below the images indicate the position of the SAM. (a) 1:1 molar CyC:PCBM blend film of $d$ $(\mathrm{PCBM})=60 \mathrm{~nm}$ average thickness. (b) Film similar to (a) after selectively dissolving PCBM. (c) Profile of the SPM scan shown in (a) perpendicular to the patterning direction. (d) FFT of the SPM scan shown in (a); next to the sharp peaks representing the grating, a blurred ring arising from droplets of different sizes are faintly visible in the background. (e) 1:2 molar CyC:PCBM blend. (f) Film of (e) after selectively dissolving PCBM. 


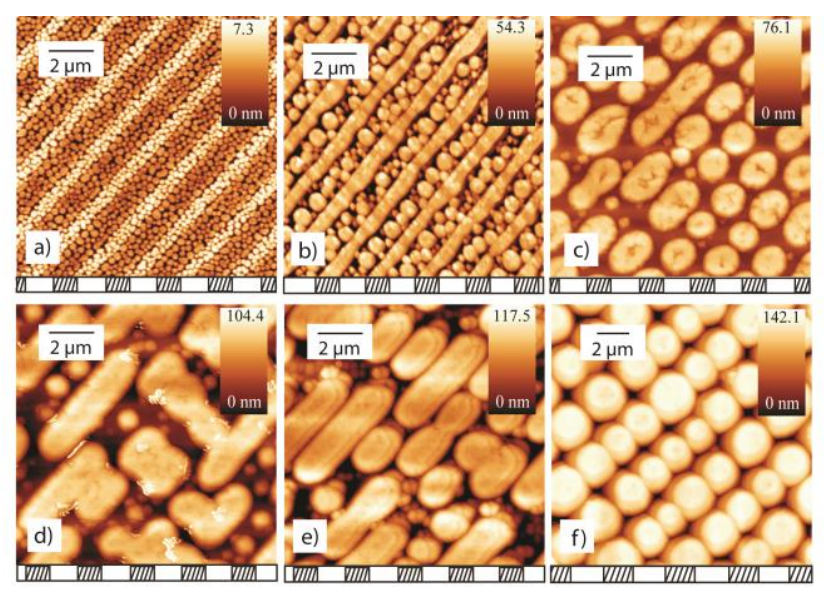

Fig. 3 SPM images of 1:1 molar CyC:PCBM blend films of increasing thickness deposited on the same surface pattern with a periodicity $\pi=1.5 \mu \mathrm{m}$. (a) Film with an average thickness $\mathrm{d}_{\mathrm{PCBM}}=60 \mathrm{~nm}$ and a corresponding natural decomposition wavelength $\lambda=0.8 \mu \mathrm{m}$ on plain gold. (b) $d_{\mathrm{PCBM}}=100 \mathrm{~nm}, \lambda=1.4 \mu \mathrm{m}$. (c) $\mathrm{d}_{\mathrm{PCBM}}=120 \mathrm{~nm}, \lambda=1.7 \mu \mathrm{m}$. (d) $\mathrm{d}_{\mathrm{PCBM}}=150 \mathrm{~nm}, \lambda$ $=2 \mu \mathrm{m}$. (e) $\mathrm{d}_{\mathrm{PCBM}}=170 \mathrm{~nm}, \lambda=2.2 \mu \mathrm{m}$. (f) $\mathrm{d}_{\mathrm{PCBM}}=250 \mathrm{~nm}, \lambda=3.2 \mu \mathrm{m}$. 


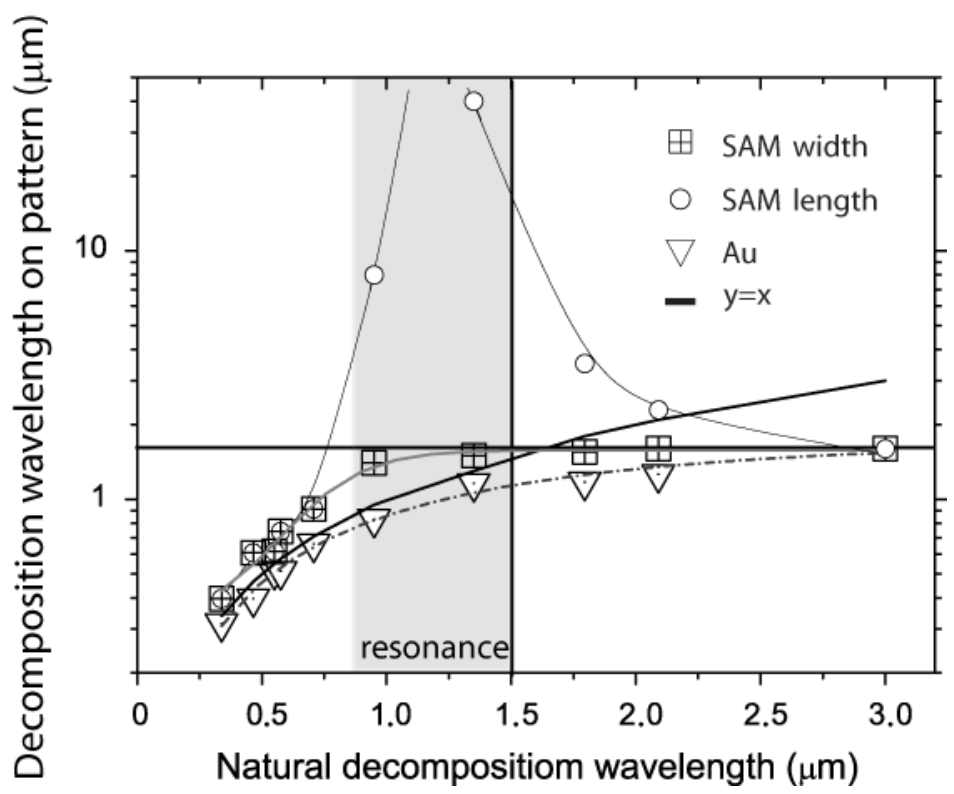

Fig. 4 Characteristic wavelengths of PCBM structures on patterned surfaces compared to the natural feature sizes observed on plain gold substrates. Wavelengths were obtained from Power Spectral Density (PSD) peaks of a large number of SPM scans. The length of the PCBM droplets along the grating direction in resonance was obtained by averaging over the length of the domains in larger SPM scans. Cross: periodicity $(1.5 \mu \mathrm{m})$ of the stripe pattern. Solid line: natural decomposition wavelength (guide to the eye only). Squares: PCBM droplet dimensions on SAM perpendicular to the grating lines. Circles: length of the PCBM droplets along the grating direction. Triangles: diameter of the droplets on gold stripes. 


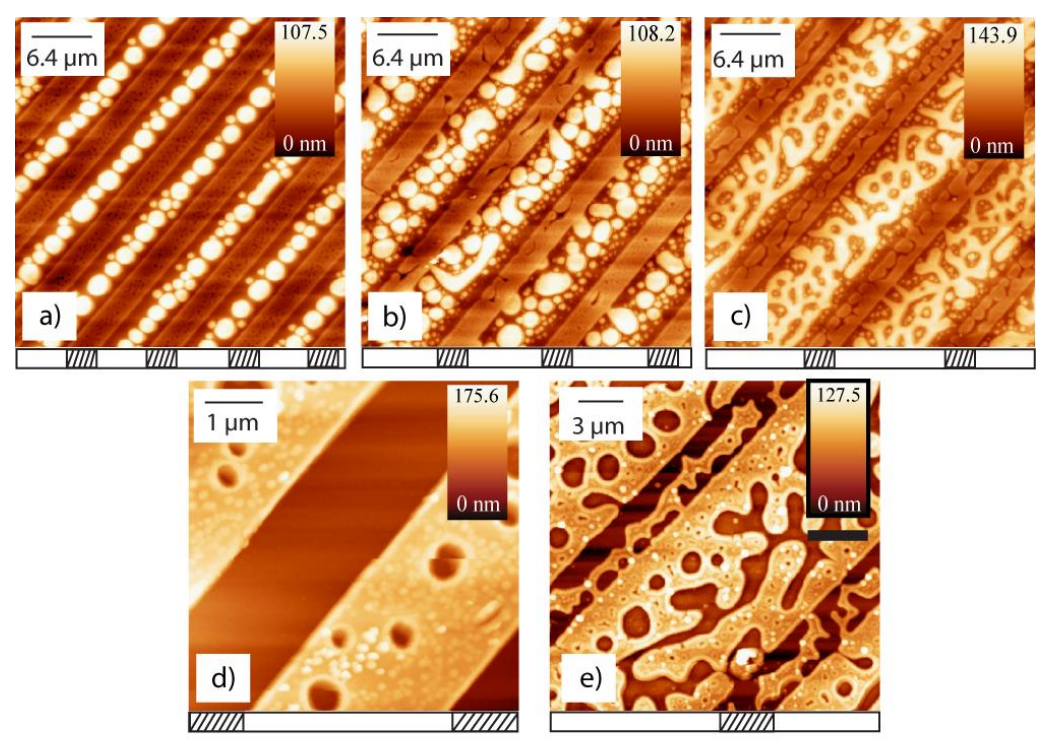

Fig. 5 SPM images of 1:1 molar CyC:PCBM blend films of $d=120 \mathrm{~nm}$ thickness on gratings with different gold stripe widths. (a) $3 \mu \mathrm{m}$ SAM line, $3 \mu \mathrm{m}$ gold line. (b) $3 \mu \mathrm{m}$ SAM line, 6 $\mu \mathrm{m}$ gold line. (c) $3 \mu \mathrm{m}$ SAM line, $8 \mu \mathrm{m}$ gold line. (d) On-resonance morphology corresponding to (a) after removal of PCBM. e) Under-resonance morphology corresponding to (c) after removal of PCBM. 
a)

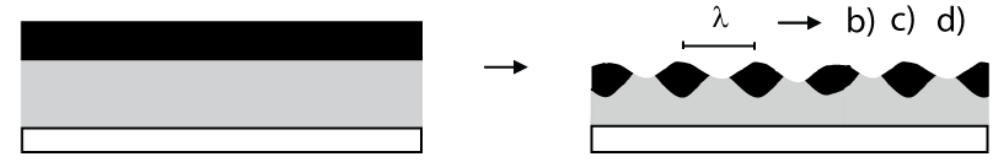

b)

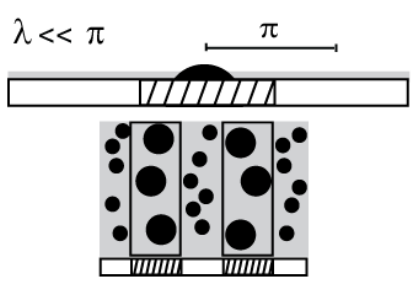

c)

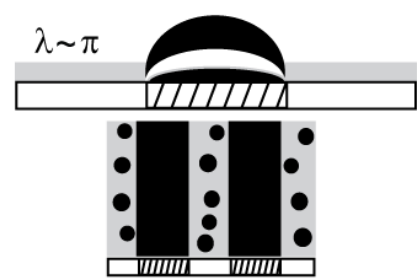

d)

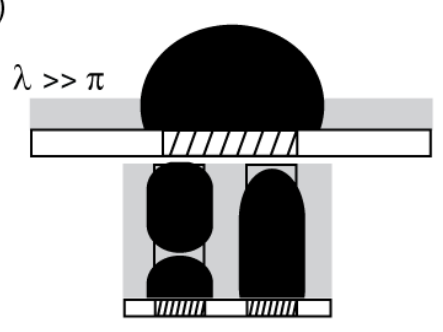

e)

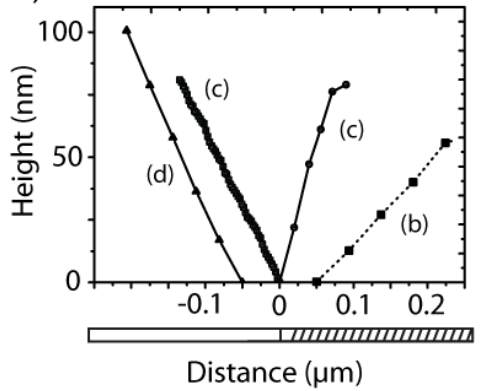

Fig. 6 Visualization of the liquid-liquid dewetting process and the contact line pinning. (a) In an early stage of the spin coating process, the solution separates into a bilayer; upon film thinning, the PCBM layer breaks up and droplets float on a cyanine layer; lenses form during solvent evaporation. Depending on the ratio of the natural decomposition wavelength $\lambda$ to the pattern periodicity $\pi$, different morphologies are obtained. (b) For small wavelengths $\lambda$ and droplet volumes, single PCBM lenses cover the SAM under a small contact angle (underresonance). (c) For the situation $\lambda \leq \pi$ (resonance), pinning of the contact line to the SAM $\mathrm{Au}$ interface extends the range of possible contact angles to $\Theta_{\mathrm{SAM}}<\Theta<\Theta_{\mathrm{AU}}$. (d) For larger droplets, at $\Theta=\Theta_{\mathrm{AU}}$, the contact line will detach from the SAM - Au interface and the droplets move onto the Au surface (over-resonance). (e) Slopes of the CyC-PCBM interface at the substrate were extracted from cross-sections of SPM data for droplets formed in under(b), on- (c) and over-resonance (d) conditions. For PCBM contact angels $\Theta<90^{\circ}, \mathrm{CyC}$ was selectively removed from the sample, for $\Theta>90^{\circ}$, PCBM was selectively removed from the sample. 


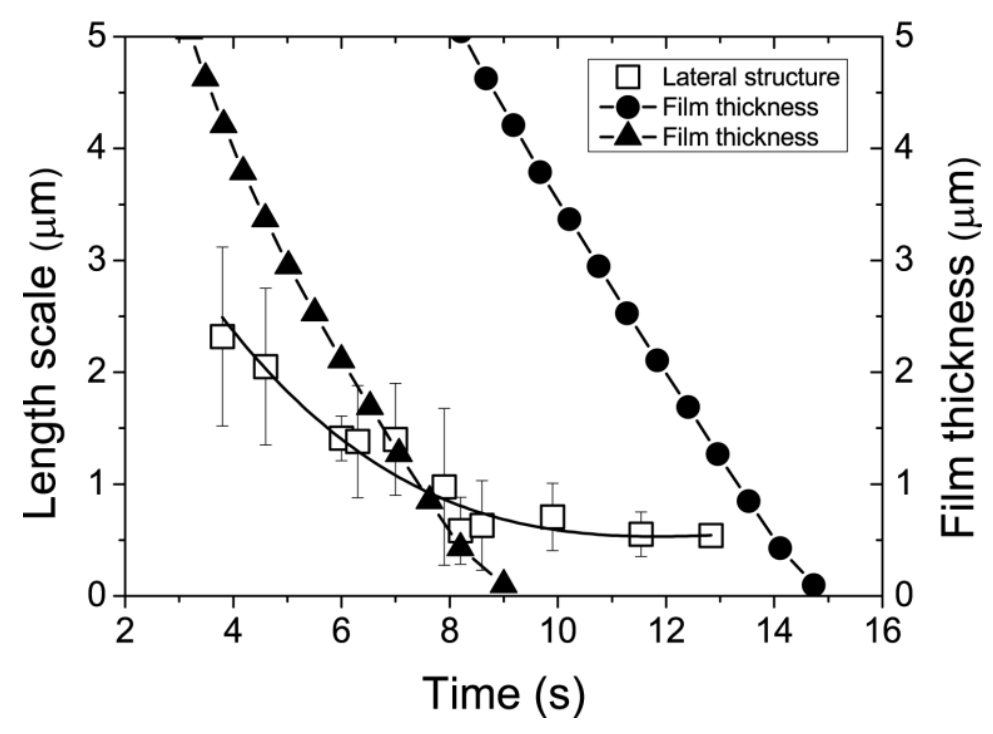

Fig. 7 Time evolutions of the in-plane characteristic length scale of PCBM domains and of the film thicknesses. Length scales were obtained from PSD of films that evolved during different time intervals, but resulted in films of the same thickness. Film thickness evolutions were extracted from time resolved laser reflectometry during coating and examples are shown for two films spin coated at $500 \mathrm{rpm}$ or $1000 \mathrm{rpm}$; all films in this work were spin coated in this speed interval. 